\title{
7.2
}

\section{Los Nuevos Instrumentos Financieros de la Banca}

D. José M. ${ }^{a}$ Ayala Vargas

Director General de Banca de Empresas. BBVA

Sumario: 1. Mercado de empresas. 2. Aproximación al mercado. 3. Productos financieros. 4. Conclusiones.

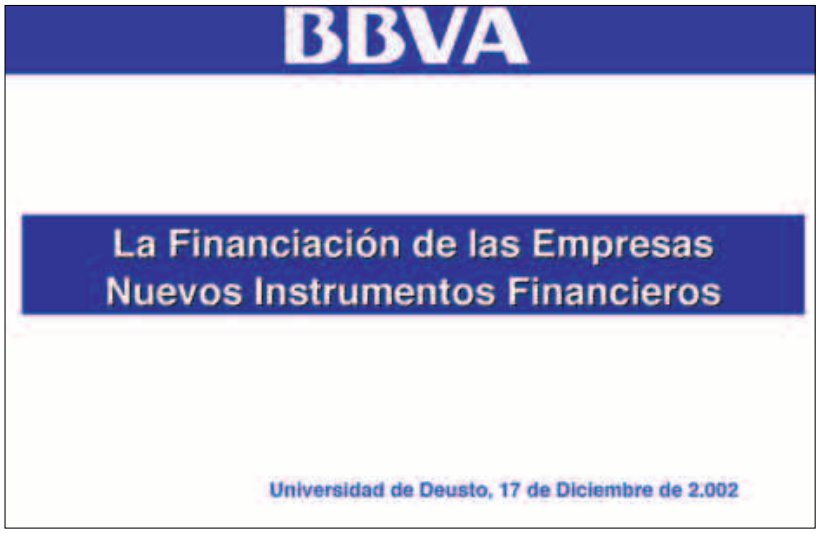

\begin{tabular}{l} 
1. EL MERCADO \\
\begin{tabular}{|l|c|c|}
\hline SEOMENTO & N TRABAJADORES & NUMERO \\
\hline CORPORATIVA & $>500$ & 1.355 \\
\hline GRANDES & $200 / 500$ & 3.276 \\
MEDIANAS & $20 / 200$ & 68.660 \\
PEOUENAS & $6 / 19$ & 181.943 \\
\hline MICRO & $1 / 5$ & 782.983 \\
\hline AUTONOMOS & & 1.631 .027 \\
\hline TOT TL & & 2.679 .244 \\
\hline
\end{tabular} \\
\hline
\end{tabular}



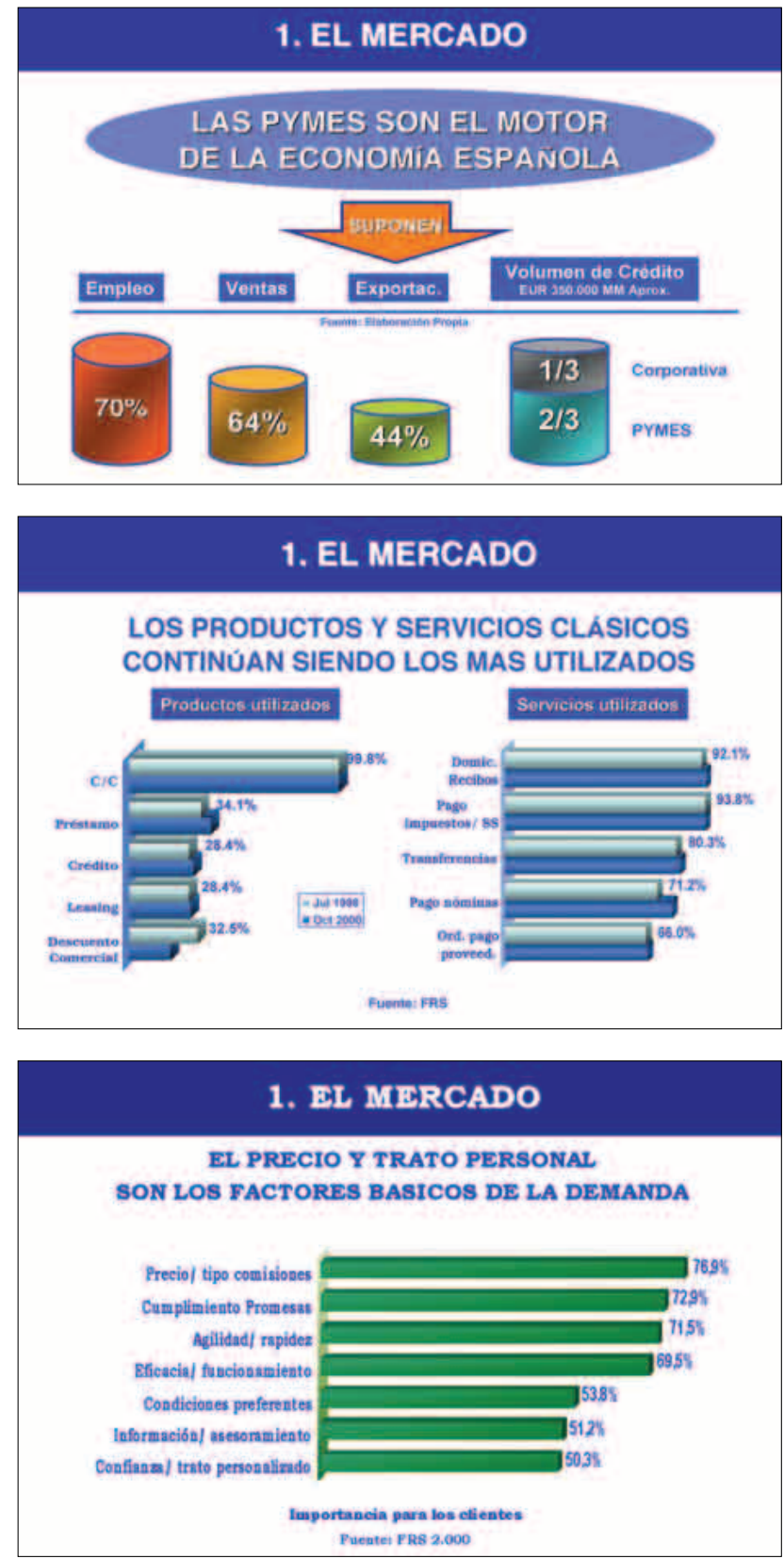


\section{EL MERCADO}

En la demanda, las tendencias estan definidas

$\triangle$ Concentración de proveedores financieros

124 Mayor exigencia de eficacia y profesionalidad en los proveedores financieros.

MA Aumento del consumo de productos financieros de mayor valor añadido

DS Fuerte crecimiento en ta utilización de canales de "banca electrónica/internet"

\section{EL MERCADO}

\section{$\triangle$ Concentración de proveedores financieros}
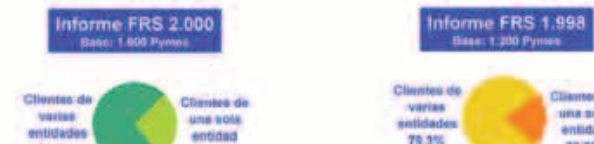

nowe

$26.3 \mathrm{~W}$
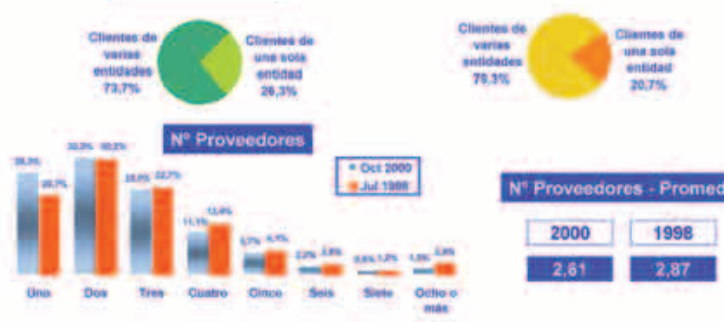

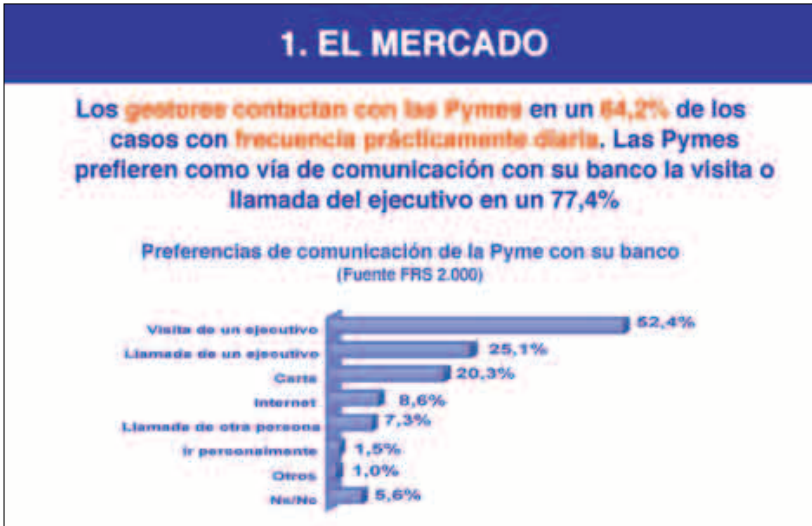




\section{EL MERCADO}

La Ofieina sigue siendo el canal más utilizado para la contratación y negoeiación de productos y servieios, aunque está creciendo de forma importante la utilización de INTERNET, especialmente como canal de información

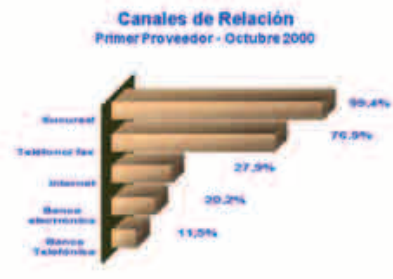

Operaciones/Consultas en internet

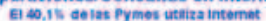

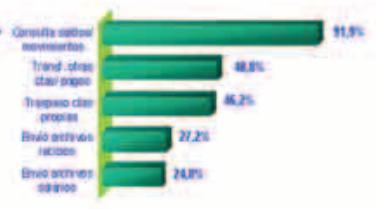

\section{APROXIMACIÓN AL MERCADO}

\section{EL MODELO DEBE RESPONDER A:}

- Elevado nivel de exigencia de servicio

- Trato personalizado

- Capacidad de interlocución

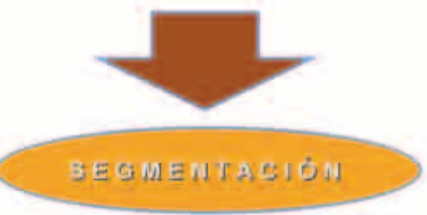

\section{APROXIMACIÓN AL MERCADO}

REDES ESPECIALIZADAS

MISION GESTION INTEGRAL DEL CLIENTE

A Corporativa

A. Empresas/Pymes

OBJETIVOS

LIDERAZGo - Participación

- Rentabilidad

- Calldad Servicio

MODELO DE GESTION , Cartera pereonalizada

- Gestor responsable de la relación del cliente con el Banco 


\section{APROXIMACIÓN AL MERCADO}

\section{MODELO DE NEGOCIO}

- pilares basicos

$\rightarrow$ Elevado nivel de delegación/responsabilidad individual.

$\rightarrow$ Sistemas de establecimiento y seguimiento de objetivos

$\rightarrow$ Valoración Individual de resultados

- FACTORES caiticos

$\rightarrow$ Valoración y seguimiento del riesgo. Pating corno elemento diferenciador de gestión y precio.

$\rightarrow$ Mejora de los indices de eficiencia

\section{APROXIMACIÓN AL MERCADO}

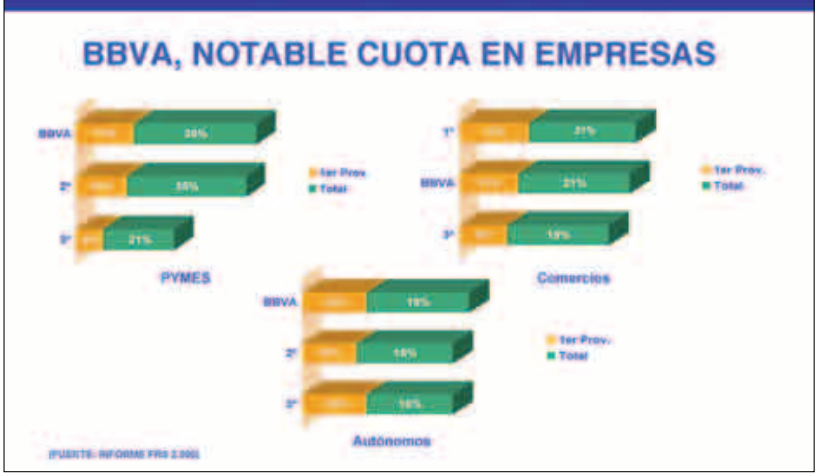

\section{LOS PRODUCTOS FINANCIEROS}

LA DEMANDA EXPEAIMENTA CIERTA EVOLUCION

- TIPOLOGIA DE PRODUCTOS

9. CLÁ́sICOS DE LA FINANCIACIÓN

Financiacion Extema ns. Fondos Propios Criterios de precio diferencial

9 GESTION DE BALANCE

Servicie, comoditad y mejors de ratios hnantieros

is REDUCCIÓN DE RIESGOS

Cobertura det nespo de interbs $y$ de cambio

- NUEVAS PROPUESTAS

Vendor Finance

Project Finance

Pasarelas de pages

E-business

Mecica de papo vinuates

Muevos Canaies. 


\section{LOS PRODUCTOS FINANCIEROS}

Productos de gestión de balance

\section{FACTORING}

- Es un instrumento financiero basado en la cesión de créditos derivados de la venta de bienes o prestación de servicios

- La cesión del crédito comercial a favor de la compania de factoring. puede comportar servicios de:

- Administración

- Gestión de cobro y

- Financiación, con o sin cobertura del riesgo

\section{LOS PRODUCTOS FINANCIEROS \\ Productos de gestión de balance}

\section{FACTORING}

- Permite anticipar el cobro de las cantidades que el cliente haya facturado.

- Anticipo no sujeto a ITP.AJD (Timbre)

- Posibilidad de cubrir hasta el $100 \%$ el riesgo de insolvencia de sus clientes, Esta modalidad se denomina Factoring sin recurso.

- Mejora su gestión de tesoreria ya que posibilita la simpilificación de los flujos de caja.

\section{LOS PRODUCTOS FINANCIEROS Productos de gestión de balance}

\section{CONFIRMING}

- Servicio de administración de pagos ofrecido a grandes clientes pagadores

- BBVA se compromete a efectuar el pago directo de las facturas del cliente a sus proveedores, que pueden o no anticipar el cobro

- BBVA emite carta de confirmación del pago a los proveedores

- Si anticipamos el pago, es sin recurso contra el proveedor $=$ rlesgo de crédito con el cliente 


\section{LOS PRODUCTOS FINANCIEROS \\ Productos de gestión de balance}

\section{CONFIRMING}

- El cliente negocia condiciones competitivas de anticipo a sus proveedores, que a su vez puede capitalizar at negociar con ellos los términos de su compra, y simplifica sus procesos administrativos

- El anticipo del proveedor no tributa ITP.AND (Timbre) y le permitte obtener liquidez inmediata sin deuda contingente (no hay recurso contra êl)

- El Banco obtiene nuevos clientes (los proveedores)

3. LOS PRODUCTOS FINANCIEROS
Productos de gestión de balance

\section{LOS PRODUCTOS FINANCIEROS Productos de gestión de balance}

\section{RENTING}

- La financiación no figura en Balance

- Es un gasto fiscaimente deducible

- El bien tampoco queda reflejado en al activo, por to que se mejora el fondo de maniobra, el RoA y los ratios de solvencia

- Permite afrontar las necesidades de inversión vía presupuesto de gastos, sin recurrir a endeudamiento.

- Al ser la renta constante por todo el periodo, convierte en costes lijos y conocidos, otros variables y/o desconocidos.

\section{Asociación Internacional}




\begin{tabular}{|c|}
\hline $\begin{array}{c}\text { 3. LOS PRODUCTOS FINANCIEROS } \\
\text { Cobertura de Riesgos }\end{array}$ \\
- DE TIPO DE CAMBIO \\
- Opciones sobre divisas (FX) \\
- DE TIPO DE INTERÉS \\
- Forward Rate Agreements (FRA's) \\
- Interest Rate Swaps (IRS's) \\
- Opciones sobre tipos de interés
\end{tabular}

3. LOS PRODUCTOS FINANCIEROS
Cobertura de Riesgos
OPCIONES SOBRE DIVISAS (FX)
- Aumento de posibilidades y máxima flexibilldad de cobertura trente at
seguro de cambio clásico (a medida)
- Mediante el pago de una prima (precio) se adquiere el derecho a
comprar (call) o vender (put) una divisa a un determinado tipo de
cambio (strike) en una fecha (vencimiento) determinada
- Posibilidad de cubrir la posición a ta baja/alta y beneficiarse de
movimientos alcistas/bajistas, en función de ser importador o
exportador

\begin{tabular}{|c|}
\hline $\begin{array}{l}\text { 3. LOS PRODUCTOS FINANCIEROS } \\
\text { Cobertura de Riesgos }\end{array}$ \\
\hline EALs \\
\hline 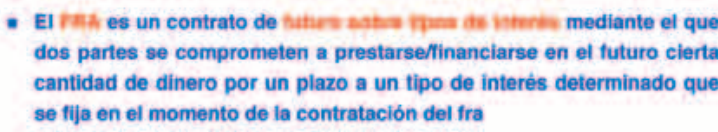 \\
\hline $\begin{array}{l}\text { - Con los iwak, las partes intercambian llujos de intereses con } \\
\text { diversas modalidades de liquidación, generalmente tipo fijo por } \\
\text { variable. Sirve para cubrirse ante las expectativas en el movimiento de } \\
\text { tipos o para mejorar las propias condiciones de financiación. }\end{array}$ \\
\hline
\end{tabular}




\section{LOS PRODUCTOS FINANCIEROS Cobertura de Riesgos}

\section{OPCIONES SOBRE TIPOS DE INTERES}

- CAP: Seguro sobre tipos de interés. Mediante el pago de una prima nos garantizamos no pagar más de un interés $x$ por nuestra financiación. La diferencia la pagará la contraparte.

- FLOOA: Garantia de rentabilidad minima. Por el pago de una prima nunca cobraré menos de un interés X' por mi inversión.

- collapi Compra de un Cap financiada con la venta de un Floor. Por mi deuda nunca pagaré más de XN a cambio de no pagar menos

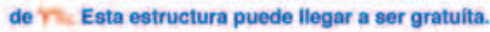

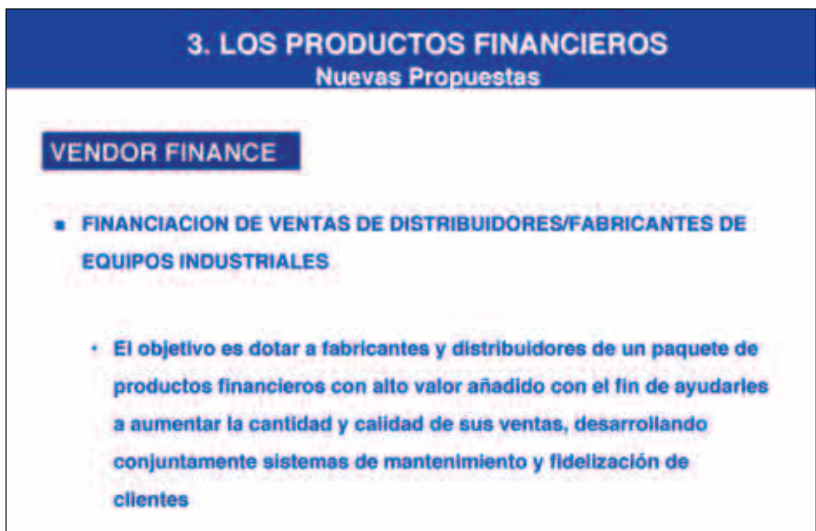

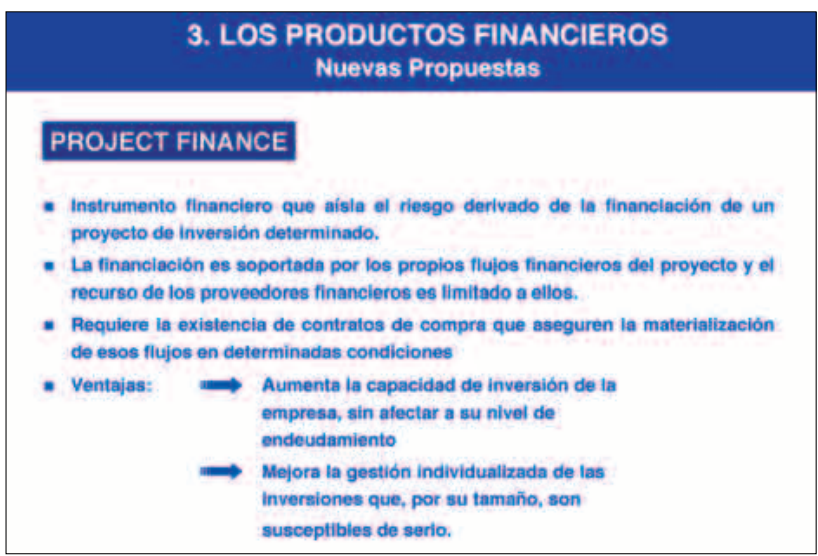

\section{Asociación Internacional}




\section{LOS PRODUCTOS FINANCIEROS}

Nuevas Propuestas

\section{PASARELAS DE PAGOS}

- Plataformas que ofrecen la posibilidad de dar a las empresas servicios de facturación electrónica, integrándolos automáticamente con servicios de transferencias, confirming, anticipos de confirming y de crédito, medios de pago virtuales...

- Estos servicios se ofrecen via internet a las empresas, independientemente de que sus transacciones se hayan realizado en un portal tipo marketplace, en su propio portal transaccional o en su relación off-line con clientes y proveedores

- Este servicio on-line también ofrece funcionalidades de generación, visualización, contirmación, negociación y modificación de facturas

\section{LOS PRODUCTOS FINANCIEROS \\ Nuevas Propuestas}

\section{E-BUSINESS}

- Portales y Marketplaces

- Facllitar la posibilidad de contratación electrónica entre proveedores y clientes, realizando tas compras a través de Internet

- Se facilita el acceso a una gran cantidad de clientes (acceso a mercado) 0 a proveedores (optimización del aprovisionamiento), con lo que se amplia cómodamente la oferta o demanda de productos

- Posibilidad de realizar negociaciones o subastas de pedidos a través de Internet

- Se optimiza el tiempo y costes derivados de una negociación, se agiliza el proceso de compra y se simplifica la carga administrativa

- Importante relación con medios de pago virtuales

\begin{tabular}{|l} 
3. LOS PRODUCTOS FINANCIEROS \\
Nuevas Propuestas \\
\hline MEDIOS DE PAGO VIRTUALES \\
- Variantes sobre tarjeta E-Purchasing (P-card): \\
- Medio de pago on-line entre distribuidor y clientes \\
- De débito o crédito \\
- Sin plástico tísico. (Solo un número y fecha de caducidad) \\
- Solo vállda para el pago a determinado/s proveedor/es \\
- Posibilidad de estabiecer límites por linea de compra \\
- Posible aplazamiento del pago \\
- El proveedor puede ser el emisor de lá tarjeta
\end{tabular}



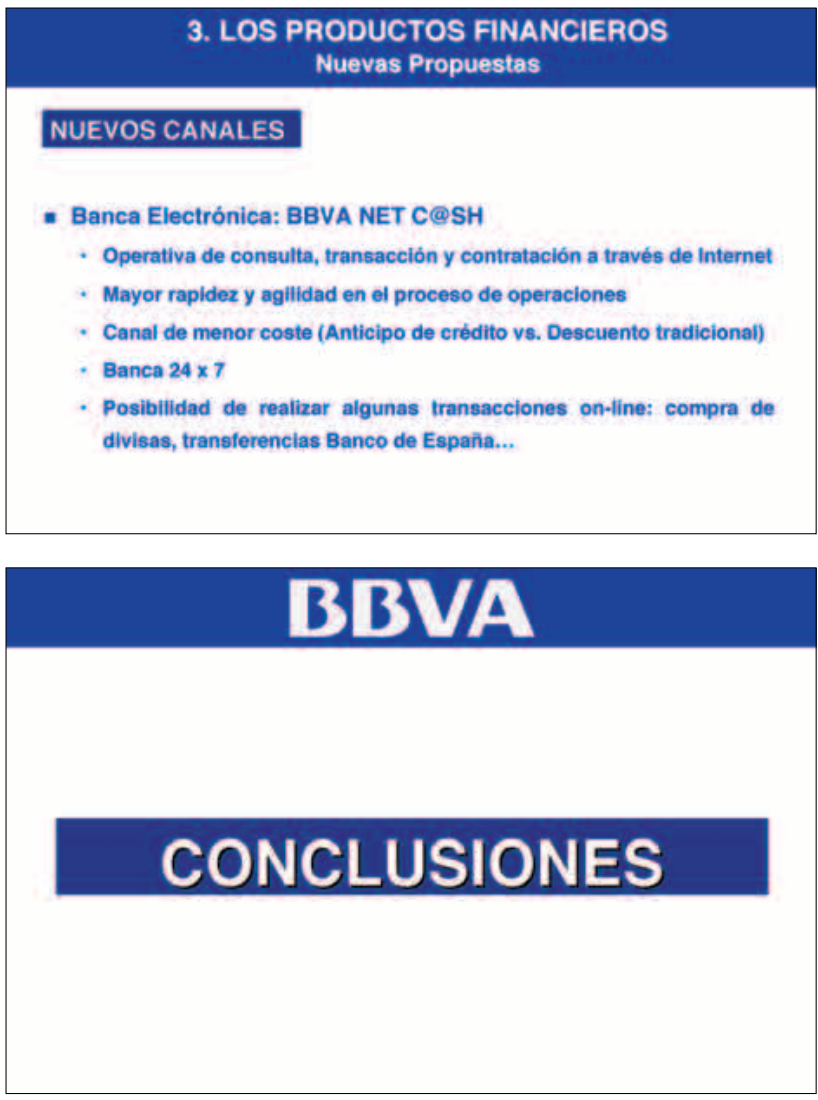

\begin{tabular}{|l|}
\hline \multicolumn{1}{|c|}{ Conclusiones } \\
\hline Financiación de Empresas: \\
Un mercado especifico \\
Las características de la demanda, modelo \\
de relación y productos hacen de la \\
financiación de empresas un negocio \\
bancario especifico
\end{tabular}




\section{Conclusiones}

El Modelo Bancario de Empresas

\section{Gestión de la oferta}

i. Las posibilidades de conocer una empresa permiten precalificarla (RATING) para la oferta de crédito

Ii Una red entrenada y experta es clave en la relación e imprescindible para delegar atribuciones de riesgo.

\section{Conclusiones}

Los Productos más utilizados: los clásicos

\section{La Banca evoluciona}

Las lineas de trabajo propuestas implican al proveedor financiero en los procesos de cobros y pagos de la empresa

internet abre un amplio campo de posibilidades de colaboración 